\title{
An analysis of transportation networks in Baoding city
}

\author{
Jiahong Xu \\ School of North China Electrical Power University, Baoding 071003, China \\ 565902301@qq.com
}

\begin{abstract}
With the improvement of living standard,more and more families tend to own their vehicle,which raise the traffic pressure in city of China and make the pollution more serious such as haze.In order to alleviate these pollution,necessary method should be taken to limit the motor vehicle,and at the same time citizen will be affected.This paper propose a model to express and evaluate the convenience of the public transit.
\end{abstract}

Keywords: network model,public transportation network.

\section{Introduction}

With the development of economic.more people tend to buy car that cause the traffic heavier and the pollution more serious.In order to alleviate these pollution,it's necessary to take method to limit the motor vehicle.However,reducing the use of personal vehicle may bring inconvenience.Therefore,it's needful to improve the service level of urban public transport for releasing the pressure of traffic and reducing the impact of the restriction on the people's travel.In this order,we need to carry out the data statistics of public transportation and evaluate convenience.In this paper,we present a model for the public transportation and have analysis of it in Baoding.To get a general evaluation, we need to figure the follow: bus station network, bus transfer network, bus lines network.Here the bus station network reflects the connection between adjacent stations. The bus transfer network reflects the link between the various sites in the entire public transport network, and the lines network reflects the links between the various bus lines.And then gain the three network characteristics of the parameters : Degree, Shortest Path distance, Clustering coefficient.

\section{Method:}

In this paper,we assume that the following condition are true:

2.1 The distances between the sites are equal.

2.2 The speed of the bus is not affected by traffic congestion, and it is a constant motion when driving.

2.3 The bus station network abstract is the undirected diagram.

2.4 Public security lines in the node that the bus lines.

The parameters and meanings in the model are as follows:

$\mathrm{V}$

$\mathrm{E}$

$\mathrm{d}_{i j}$

G
The point set in network model

The edge set in network model

The shortest distance between node i and node $\mathrm{j}$

Network diagram 
$\mathrm{M}$

$\mathrm{N}$

$\mathrm{k}$

$\mathrm{P}(\mathrm{k})$

$\mathrm{L}$

$C_{\mathrm{i}}$
The number of nodes in the network model The number of edges in the network model The degree of each node The distribution of degrees Average shortest distance

The clustering coefficients of all nodes

Here:

(1) Average shortest path distance

The shortest path distance between two nodes $i$ and $j$ in network $G$ is defined as the number of edges of the shortest path that connecting in two nodes.For an undirected network,the average shortest path distance can be defined as follow:

$$
l=\frac{1}{N(N+1)} \sum_{i, j \in V(i \neq j)} d_{i j}
$$

(2) The Clustering coefficient

Assuming that a node $\mathrm{i}$ in network $\mathrm{G}$ is connected to $\mathrm{k}_{i}$ nodes with $\mathrm{k}_{i}$ edges. These $\mathrm{k}_{i}$ nodes are called the neighbor of node i.Obviously,this network may have up to $\mathrm{k}_{i}\left(\mathrm{k}_{i}-1\right) / 2$ edges. The ratio of the number of edges $E_{\mathrm{i}}$ to the total number of possible edges between $k_{i}\left(k_{i}-1\right) / 2$ is defined as the clustering coefficient of node $i$,and the average of the clustering coefficients of all nodes in the network is the coefficient of class:

$$
C_{i}=\frac{E_{i}}{k_{i}\left(k_{i}-1\right) / 2}=2 E_{i} / k_{i}\left(k_{i}-1\right)
$$

The acquisition of clustering coefficient:

Step 1: Get the neighbor matrix

In the case of an ordinary public transportation station network,two bus stations i and $\mathrm{j}$ have the same bus line name, then the two stations $\mathrm{i}, \mathrm{j}$ are connected, adjacency matrix The element $\mathrm{M}(\mathrm{i}, \mathrm{j})=1$, otherwise $\mathrm{M}(\mathrm{i}, \mathrm{j})=0$.

Step 2: Calculate the degree of the node

Step 3: Calculate the shortest path distance

Use Floyd algorithm to calculate, which is the basic idea:

1) Define a matrix $D$ to record the information of the inserted point, $D(i, j)$ denote the point from node $i$ to node $j$, and initialize $D(i, j)=j$.

$(I, j)=\min (M(i, j), M(i, j)+M(k, j))$, and the distance between the interpolated points and the original distance, If the value of $M(i, j)$ becomes smaller, then $D(i, j)$ $=\mathrm{k}$. 
3) in D contains the shortest path distance information, according to this information to calculate the shortest path distance.

Step 4: Node clustering factor

1) Calculate the clustering coefficients of $i$, and delete all nodes that are not connected to i.

2) Determine the number of nodes connected to point $i$

3) Determine the number of edges of all nodes connected to the i point

4) Calculate node clustering coefficient according to (2).

Bus line optimization model:

Public transport occupies a large proportion in urban passenger transport. Improve the operational efficiency of public transport is conducive to improving the city's passenger transport effect. Hence,we need to consider the needs of bus passengers to meet their travel shortest time, taking into account the operating costs of bus companies. Here we set up a public transport network optimization model.

In the case of a constraint, the objective function is the sum of the operating costs and the user's cost, which determine which bus lines are route and how the starting frequency of each bus line is optimization.Here the user's cost is a function of the total travel time, and the operator's cost is mainly determined by the operating mileage of the vehicle under the specific configuration conditions. Constraints include: the range of departure intervals, the limits of vehicle capacity, fleet size and so on. Build the model as follows:

$$
\begin{gathered}
\text { Min: } \mathrm{Z}=c 1 \sum_{i, j=1}^{n} d_{i j} t_{i j}+c 2 \sum_{k \in R_{S R}} f_{k} t_{k} \\
\text { s.t }\left\{\begin{array}{c}
f_{k} \geq f_{\min } \quad \forall k \in R_{S R} \\
l_{k} \leq l_{\text {max }} \quad \forall k \in R_{S R}
\end{array}\right.
\end{gathered}
$$

Here $d_{i j}$ is the travel demand from node $\mathrm{i}$ to node $\mathrm{j}$; $t_{i j}$ is the sum of the travel time of node $\mathrm{i}$ to node $\mathrm{j}$ (including vehicle time, waiting time and transfer loss time); $\mathrm{f}_{k}$ is the minimum starting frequency of the bus line of $k$; $t_{k}$ is the whole run time of bus line $\mathrm{k} ; \mathrm{f}_{\min }$ is the minimum starting frequency of bus line; $\mathrm{l}_{k}$ is the full load rate of bus line $\mathrm{k}$; C is the rated passenger capacity of the bus; $Q_{k}^{\max }$ is the largest section of bus line traffic; $R_{\mathrm{sr}}$ is a collection of bus lines; $C_{1}$ is the conversion factor that converts the user's travel time into the user's travel expense. $C_{2}$ is the conversion 
coefficient converted into bus operating costs by bus mileage. $\mathrm{n}$ is the number of network nodes.

$$
l_{k}=\frac{Q_{k}^{\max }}{f_{k} \cdot C}
$$

\section{Result}

(1)The characteristics of bus network in Baoding A.node degree and degree distribution

The bus network model of the bus station indicates the number of bus stations that a station is connected with. It can be calculated that the degree of each station in Baoding City's bus station network is 2 to 9, and the average degree is 3.3708, 3 sites connected.

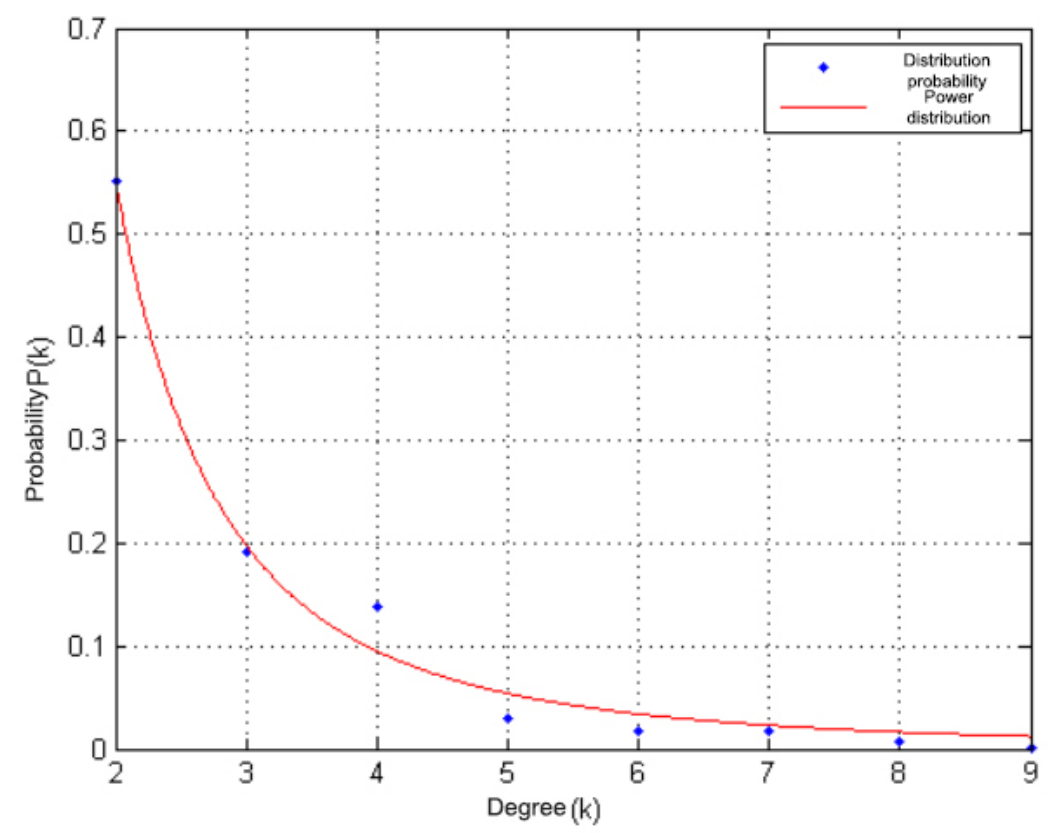

Figure 1

Figure 1 shows the probability distribution of the network degree. It can be seen that the degree of most sites is between 1 and 10, and only a few stations are greater than 6 , and these sites are hub stations.

B.The shortest path distance and the average shortest path distance

The shortest path in the bus network indicates the least edges that the minimum distance from one station to another, and the average shortest path distance is the average of the shortest path distance.The maximum path matrix is obtained by Floyd algorithm according to the adjacency matrix of Baoding city transit network.And the shortest path distance range of Baoding city bus network is $0 \sim 40$, the average shortest path distance is 17.This indicates that there is an average of 17 stops from one station to another in Baoding.The shortest distance probability distribution is shown in Figure 2. 


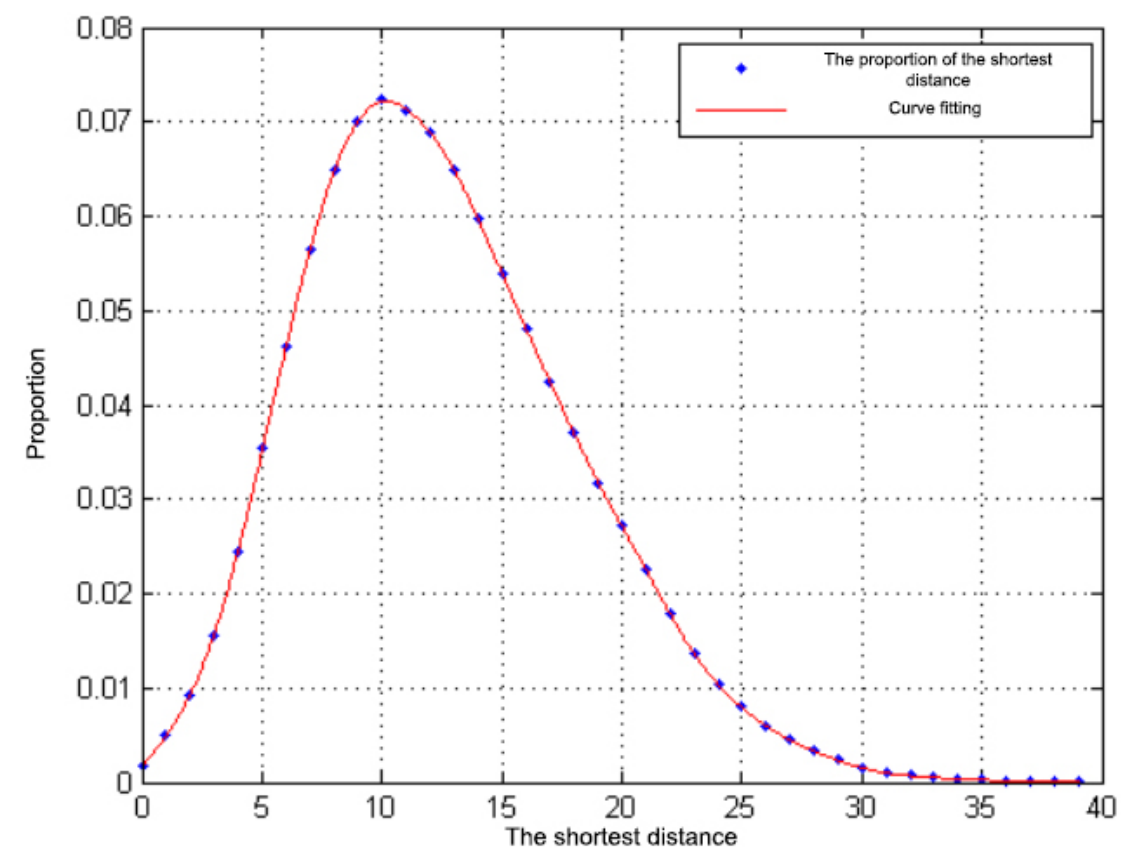

Figure 2

C The clustering coefficient

The clustering coefficient indicates the intensive situation of the bus line between the sites connected with the site.And the average clustering coefficient reflects the line density of the bus station network.The clustering coefficients for each frequency segment are shown in Figure 3.

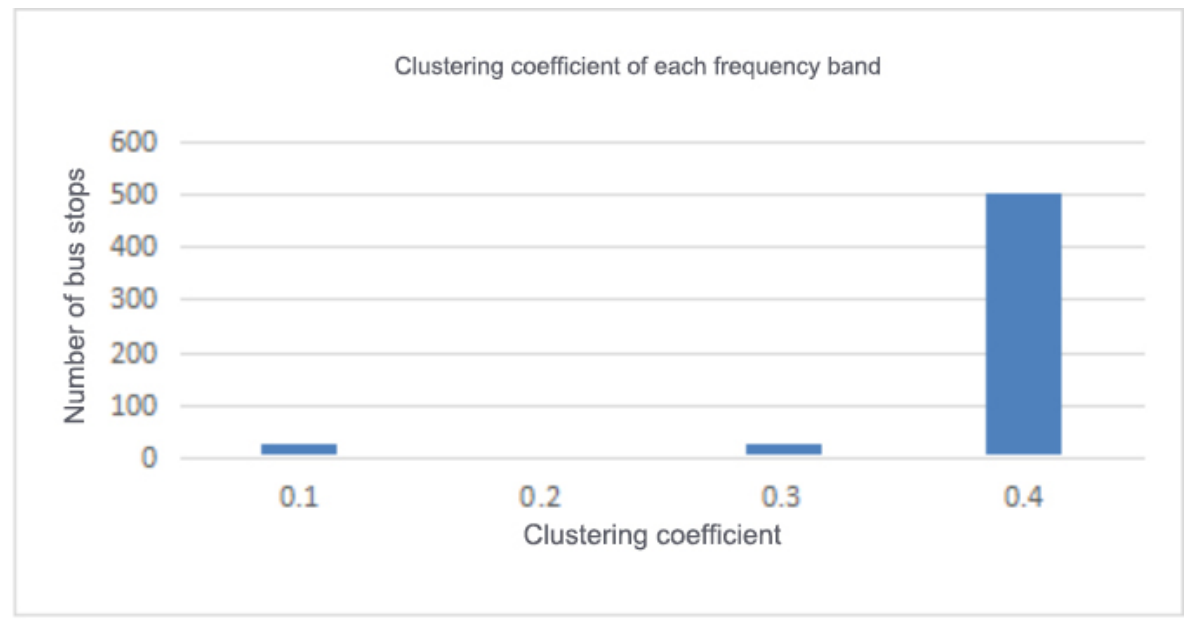

Figure 3

The clustering coefficient of most sites in Baoding city bus network is 0.4.The average clustering coefficient of the network is large, which indicate that the public security station density of Baoding is larger.

(2)The analysis on the Characteristics of Bus Transfer Network in Baoding City A.Node degree and degree distribution

The degree of the bus transfer network node indicate the number of sites that can be accessed directly from the station without other lines.And The value of the site is 
the hub of the bus network. The statistical figure shows the probability distribution map of Baoding public transport network, as shown in Figure 4.

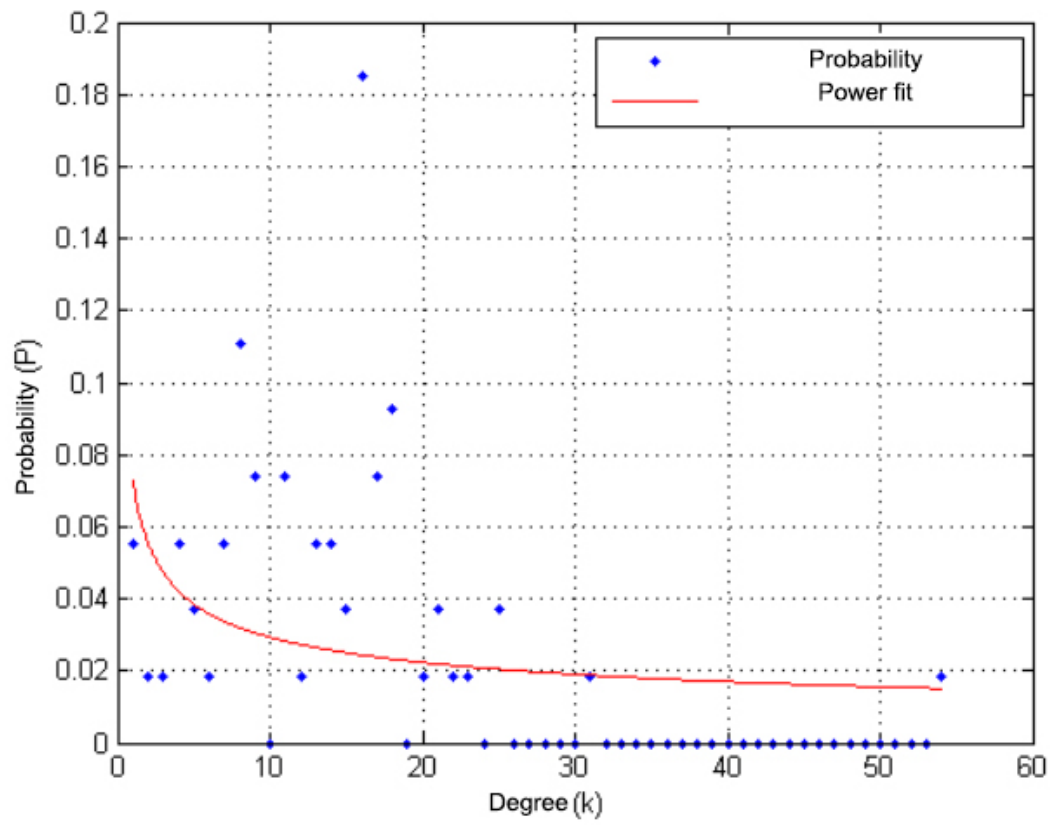

Figure 4

It is found that the distribution of node degree of bus network in Baoding is not consistent with the power distribution.

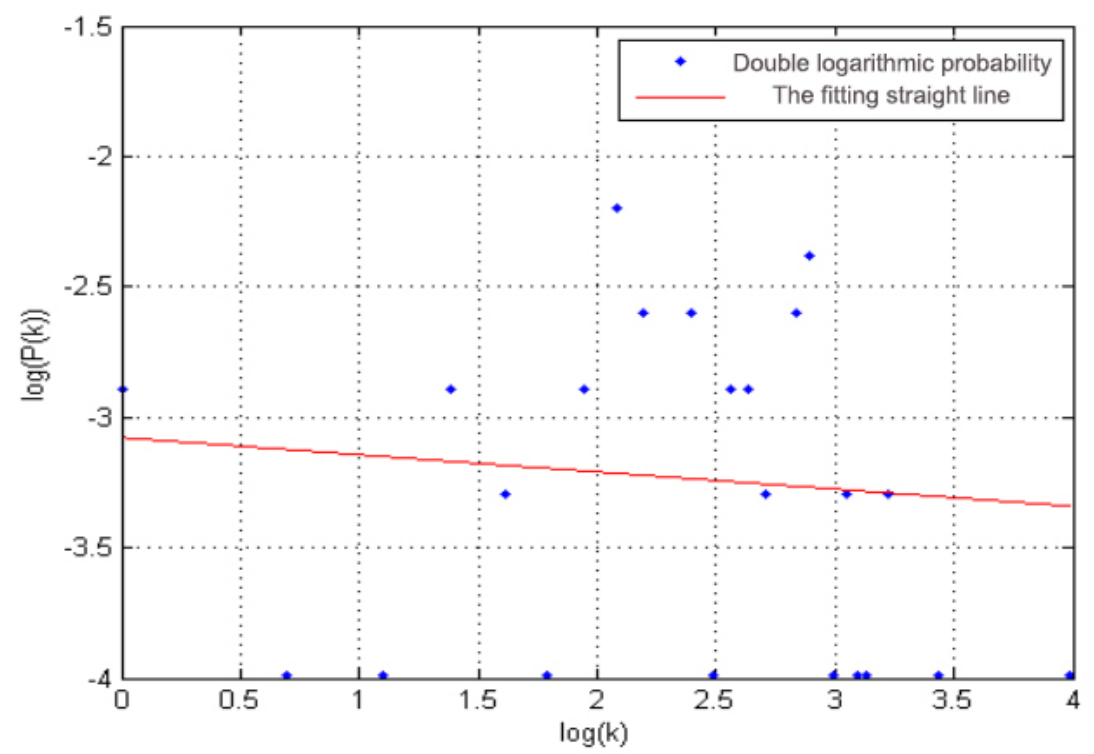

Figure 5

Figure 5 shows the degree distribution of the double logarithmic index. From the double logarithmic index,it can be clearly seen that the Baoding bus network does not have a scale.Through the statistics it can be found that most of the nodes are of the degree of 3 to 25 , a small number of sites for the 30 , while these few sites are the traffic hubs. 
B.The shortest path distance and the average shortest path distance

The shortest path matrix can be calculated by the actual adjacency matrix of the bus transit network in Baoding.And the shortest path distance distribution of Baoding transfer network is shown in Figure 6.

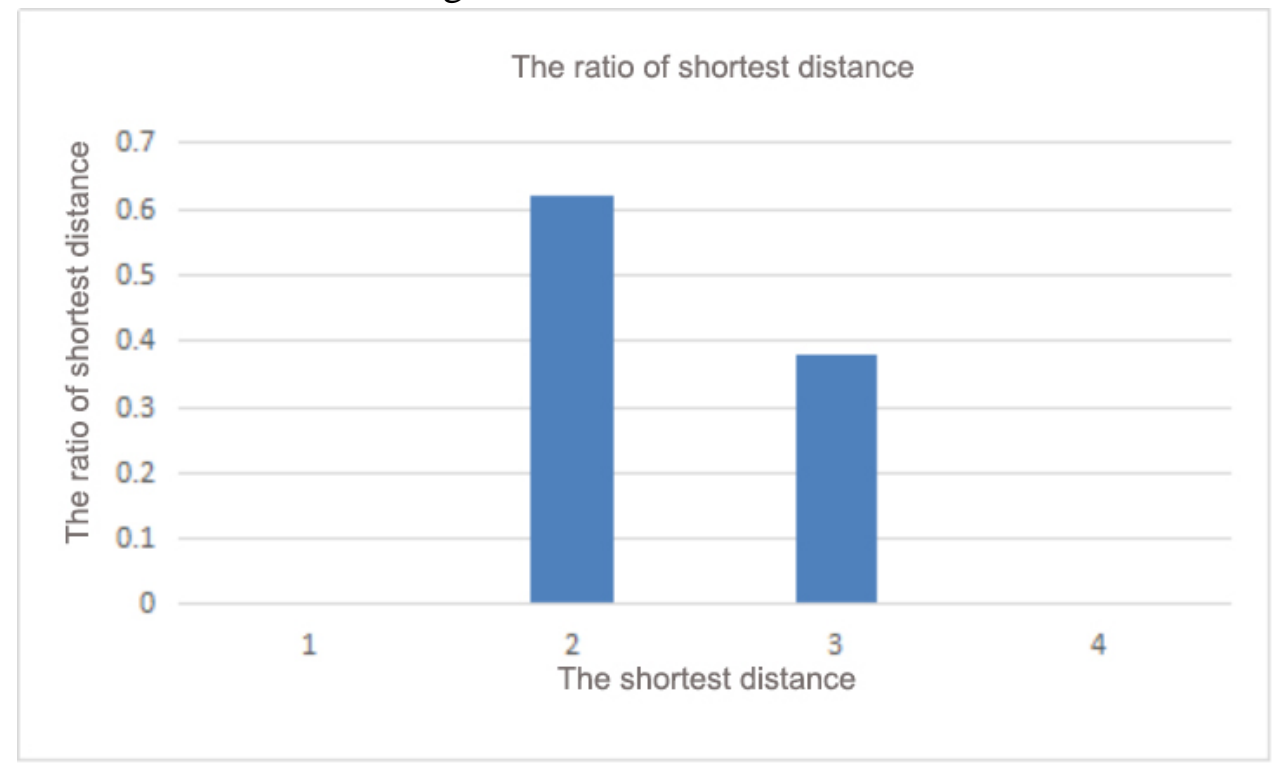

Figure 6

The shortest path distance between nodes in the bus transfer network model represents the number of times that need to be transferred from one station to another.The average number of transfers is the average shortest path distance.The average shortest path distance to the range is 2 to 3.It can be calculated that the average shortest path distance is 2.38 that is, in Baoding, it is average 1.4 times to take bus.

\section{The clustering coefficient}

The bus transfer network clustering coefficient represents the possibility of having a direct line between adjacent stations.The larger the clustering coefficient of the site, the better the transfer between the site and the adjacent site. The distribution of clustering coefficient of bus transit network in Baoding is shown in Figure 7. 


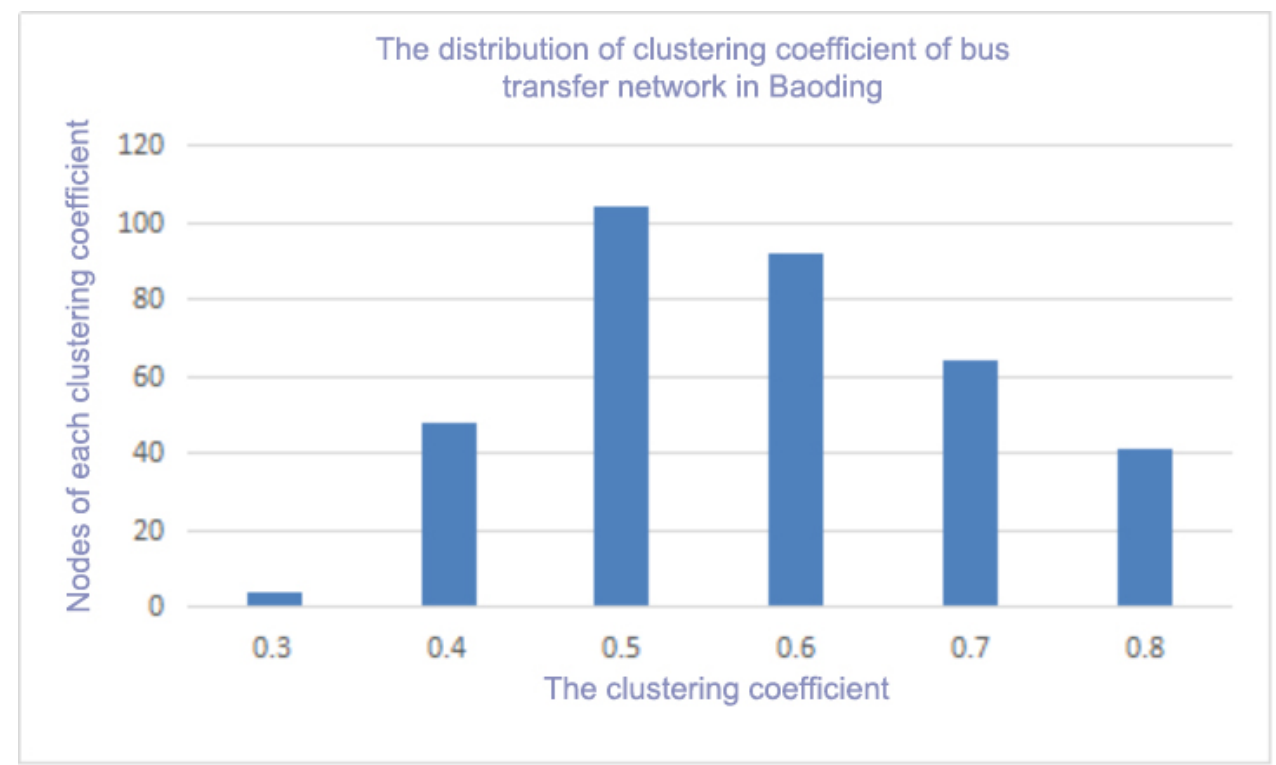

Figure 7

The average clustering coefficient can be calculated as 0.582 . The larger the clustering coefficient, the less possibility it is to have a direct line between adjacent sites.

(3) The analysis on the characteristics of bus network model in Baoding city.

A.Node degree and degree distribution

By calculating the adjacency matrix in Baoding city, we can get the degree of each node, which the range is $1 \sim 55$, and the average degree is 18 . The probability distribution of nodes is shown in Figure 4.It is shown in Figure 4 that the probability distribution of Baoding city bus network network nodes is uniform and does not conform to the power probability distribution.Figure 5 shows the distribution of the double logarithmic index.It can be obviously seen that Baoding city bus network does not have a scale.

B.The shortest path distances and the average shortest path distance

Figure 8 shows the shortest path distance distribution between the bus nodes in Baoding. 


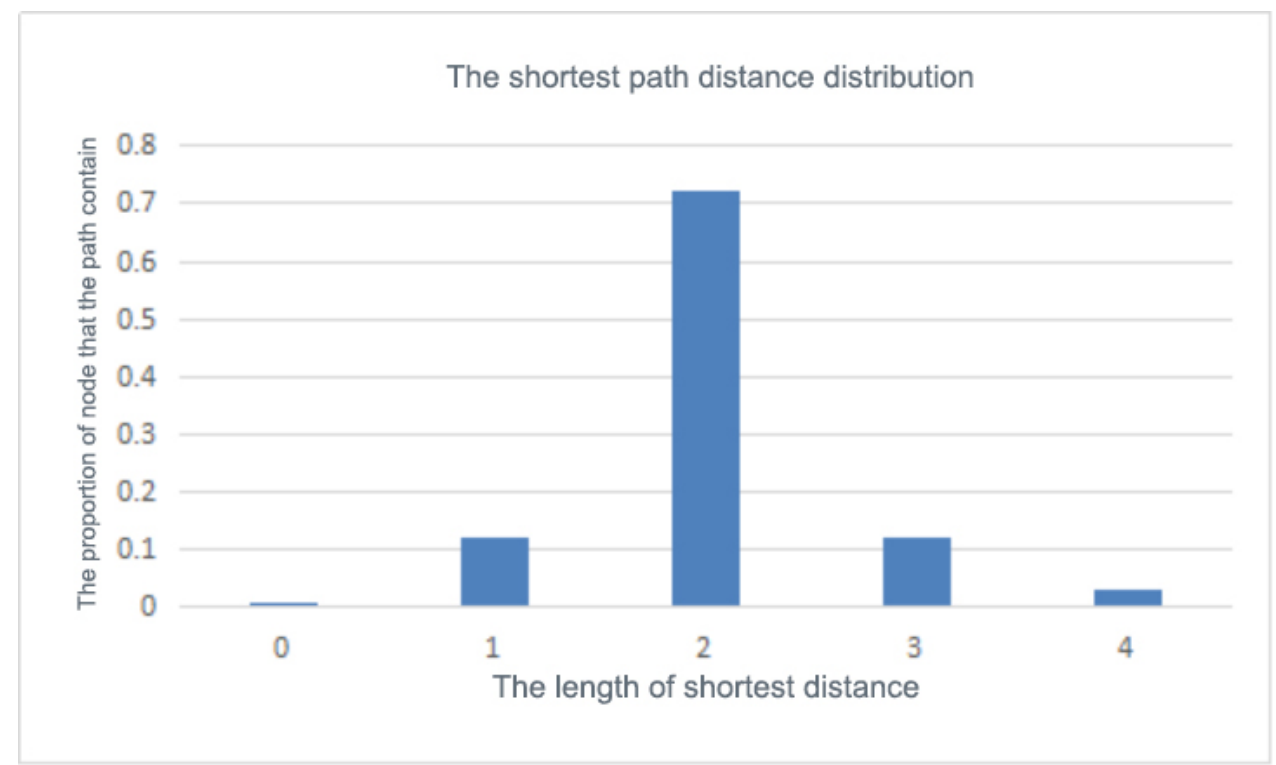

Figure 8

Figure 10 shows that the shortest path distance range is 0 to 4 , and the average shortest distance of 2.207, that is, about 1.207 times from a line transfer to another line.

C.The clustering coefficient

The distribution of the clustering coefficients in Baoding is shown as Figure 11.

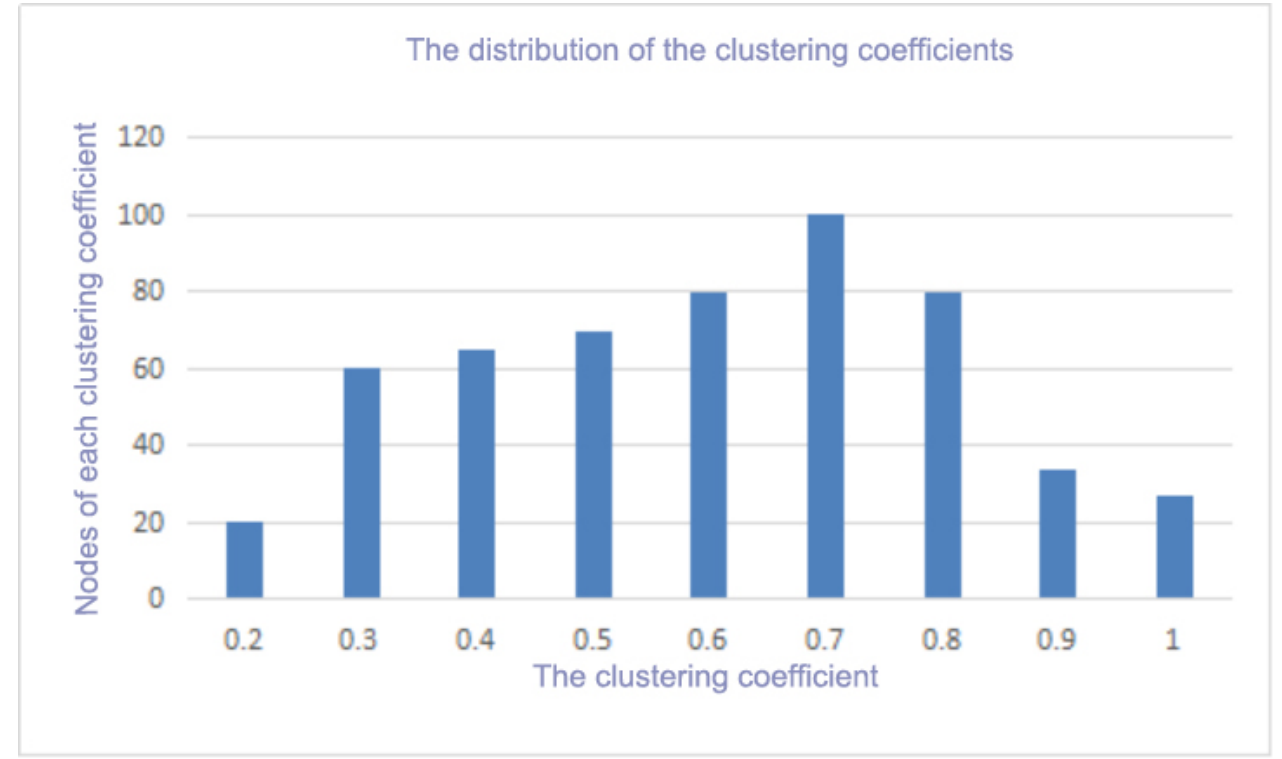

\section{Figure 9}

Figure 11 shows that the average clustering coefficient is 0.5383 .And $97.5 \%$ of the nodes is larger than 0.3 , which indicate that the probability of connection between adjacent lines is low.

\section{Conclusions}

For the evaluation model of the bus line, this paper uses the analysis method of the network model to evaluate the rationality of the bus line in Baoding city. It is found that the line condition of Baoding City is generally better.But there is still lots of problem such as a poor transfer,and the large average distance of each site. 
The model proposed in this paper does not take the traffic jam, the concrete factors in the actual practice into account. Hence,we will improve the model in the short term.

\section{References}

[1]Illiani Carro-Pérez,Hugo G. González-Hernández."Topological characterization of the public transportation complex network in Puebla City, México",IEEE,24-26 Feb. 2016,pp:113-117.

[2]Sabrina Gaito, Dario Maggiorini, Elena Pagani, Gian Paolo Rossi."Distance Vector Routing for Public Transportation Vehicular Networks: Performance Evaluation on a Real Topology".IEEE,15-17 Dec. 2009.

[3]Andor Háznagy,István Fi,András London,Tamás Nemeth."Complex network analysis of public transportation networks: A comprehensive study",IEEE.3-5 June 2015.pp:371-378.

[4]Yang Jin."Complex Network Characteristics Analysis of Beijing Public Transport Network"[D]. Beijing Jiaotong University.Dec,2012.

[5]Liu Chuang,Han Yin."Research on Intelligent Public Transport Network Optimization Method Based on Genetic Algorithm"[J].Computer Engineering and Applications.2003.pp:34,39(34):208 209.

[6]Liu Haode. "Optimization Design Theory and Realization Method of Bus Network"[D].Tongji University. 1 Jan ,2008.

[7]Si Shoukui. "Mathematical modeling algorithm and application"[M].1.National Defend Industry Press .2015. 